\title{
Lerntherapie für die Welt
}

\author{
Michael von Aster, Liane Kaufmann, Irene Corvacho del Toro, Ursula Fischer, Karin Kucian, \\ Marlies Lipka, Jens Holger Lorenz, Cordula Löffler, Marianne Nolte und Gerd Schulte-Körne
}

Ein neues Virus! Unsichtbar, unbekannt, unberechenbar, hoch ansteckend aber oft unfühlbar, potenziell lebensbedrohlich, exponentielle Ausbreitung, Angst, Tote, chaotische Überlastung von Krankenhäusern, Totstellreflex: Lockdown! Quarantäne, Hygiene, soziale Distanz, Gesichtsmasken, zu Hause bleiben. Alles wird langsam, ruhig und sauber. Als würde die Welt stillhalten und Luftholen.

Da war doch was? Klimakrise! Aufgeklärte Schüler bleiben der Schule fern für „Fridays for Future“! Das scheint stärker aufzurütteln als die alarmierenden Zahlen und Diagramme zum Temperaturanstieg, der ja auch (noch) nicht täglich und für Jeden leiblich fühlbar ist.

Warten im Lockdown: Keine Arbeit, keine Geschäfte, keine Restaurants, keine Alltagshektik, kein Gewohnheitsrhythmus, wachsende Sorgen: Zweite Welle, Wirtschaftskrise, eintrübende Zukunft, verunsicherte Hoffnung auf Medikamente und Impfstoff. Kinder zu Hause, Langeweile, Ablenkung, wachsender Stress, Kindeswohl, Mütter wieder in der Pflicht.

Da war doch was? Gleichstellung von Frauen und Männern in Familie und Beruf? Wie es scheint, garantieren Kindergarten und Ganztagsschule primär für Frauen, weniger für Männer, berufliches Fortkommen.

KiTas und Schulen geschlossen, Homeschooling, wenn's funktioniert!

Da war doch was? Digitalpakt, Digitales Lernen, Zoom, Lernplattformen. Wie es scheint, klafft hier eine große Lücke zwischen deklarierten Absichten und tatsächlicher Umsetzung und Verfügbarkeit. Man improvisiert, so gut es geht! Bei leistungsstarken Kindern und solchen aus bildungsnahen Schichten funktioniert es besser. Manche können sogar profitieren. Kindern am anderen Ende dieses Spektrums fehlt es dagegen an Ausstattung und Unterstützung, sie driften noch weiter nach unten: Schereneffekt! Auch im universitären Bereich wird der Lehrbetrieb auf Online-Plattformen umgestellt. Ob und mit welchen Nebeneffekten das Digitale Lernen an den Universitäten funktioniert, bleibt abzuwarten. Eine aktuelle Studie an der Universität Zürich untersucht die Folgen dieser Umstellung für Motivation und Wohlbefinden der Studierenden (Sieber, 2020).
Der Lockdown wirkt: Die Viruslast sinkt, die Zahlen von Infizierten und Verstorbenen gehen zurück. Vorsichtige Lockerungen: Zuerst die Wirtschaft, die Geschäfte, der Verkehr, und zuletzt die KiTas und die Schulen, und dort zuerst die Älteren und zuletzt die Jüngsten.

Da war doch was? Dänemark! Die machen es umgekehrt und besser! Richtig, denn bei den Jüngeren gehen die noch zarten, unvollkommenen schulischen und sozialen Wissensund Fähigkeitsstrukturen wieder verloren, wenn sie zu lange keine Resonanz im täglichen Gebrauch erfahren. Bei den Älteren sind diese Strukturen schon gefestigter, Verlust ist kaum $z u$ befürchten, allenfalls verlangsamter Fortschritt. Solches Grundlagenwissen aus der Entwicklungs- und Neuropsychologie hat es scheinbar noch immer schwer, im Bildungssektor Gehör zu finden.

Der Spiegel titelt Ende April: „Schulversagen: Wie das Virus die Schwächen unseres antiquierten Bildungssystems offenlegt“, und The Guardian Mitte Mai: „Coronavirus shows us it's time to rethink everything. Let's start with education!" Erste empirische Erhebungen zu den bildungsbezogenen Folgen von COVID-19 scheinen die genannten Befürchtungen zu bestätigen und verstärken den Ruf nach tiefgreifenden Bildungsreformen (Huber et al., 2020). Unter dem Brennglas der Pandemie erweist sich unser Bildungssystem als unvorbereitet. Mehr und besser ausgebildete Lehrund Erziehungskräfte, eine bessere Verfügbarkeit und Vertrautheit mit digitalen Unterrichts- und Kommunikationsmethoden sowie mehr Räumlichkeit und geeignete Infrastruktur für Teilungs- und Fraktionierungsstrategien hätten frühzeitig sowohl mehr Betreuung und Beschulung in Kleingruppen für die Jüngeren vor Ort als auch effektiveres Homeschooling für die Älteren möglich gemacht. Und es hätte auch das Verstehen und Erlernen von achtsamen Distanz- und Hygieneregeln unterstützen und damit die Ansteckungsrisiken im Blick behalten können, von denen wir erst neuestens annehmen dürfen, dass sie in Schulen ohnehin gering sind (Viner et al., 2020).

Dies ist ein Blick von heute, einem Tag im späten Mai. Bis dieses Editorial im frühen Juli erscheint, werden wir in Sachen COVID-19 noch viel Neues dazugelernt haben. Aber soviel lässt sich doch sagen, und das ist nicht neu, 
aber zunehmend dringlich: Qualitativ hochwertige Bildung für alle benötigt endlich vorderste Priorität, denn sie verbessert die Chancen für aufgeklärtes, d.h. wissens- und wissenschaftsbasiertes Urteilen und Handeln, und ist damit auch die beste Prävention und Therapie (um nicht zu sagen Impfstoff) gegen jene gefährliche Lernstörung, die die Zukunftsbedrohungen unserer Zeit in populistischer Weise leugnet und damit das Potenzial zu einer geistigen Pandemie mit schlimmeren Folgen hat.

\section{Literatur}

Huber, S. G., Günther, P. S., Schneider, N., Helm, C., Schwander, M., Schneider, J.A. \& Pruitt, J. (2020). COVID-19 -Aktuelle Herausforderungen in Schule und Bildung. Erste Befunde des Schul-Barometers in Deutschland, Österreich und der Schweiz. Münster, New York: Waxmann.
Sieber, V. (2020, May 2). Motivation and well-being of university students in online courses during social distancing: The role of supporting the satisfaction of the basic need for relatedness. Verfügbar unter osf.io/jsa35

Viner, R. M., Russell, S. J., Croker, H., Packer, J., Ward, J., Stansfield, C. ... Booy, R. (2020). School closure and management practices during coronavirus outbreaks including COVID-19: a rapid systematic review. The Lancet Child \& Adolescent Health, 4, $397-404$.

\section{Prof. Dr. Michael von Aster}

DRK Kliniken Berlin I Erziehung und Bildung GmbH

Zentrum für Schulische und Psychosoziale Rehabilitation (ZSPR)

Spandauer Damm 130

14050 Berlin

Deutschland

m.aster@drk-kliniken-berlin.de 